\title{
Potassium fluoride and carbonate lead to immediate cell failure in po- tassium-ion batteries
}

\author{
Andrew W. Ells, Richard May, Lauren E. Marbella* \\ Department of Chemical Engineering, Columbia University, 500 W 120th St, New York, NY 10027
}

\begin{abstract}
While Li-ion is the prevailing commercial battery chemistry, development of batteries using earth abundant alkali metals (e.g., $\mathrm{Na}$ and $\mathrm{K}$ ) alleviates reliance on Li with potentially cheaper technologies. While electrolyte engineering has been a major thrust of Li-ion battery (LIB) research, it is unclear if the same electrolyte design principles apply to K-ion batteries (KIBs). Fluoroethylene carbonate (FEC) is a well-known additive used in Li-ion electrolytes, because the products of its sacrificial decomposition aid in forming a stable solid electrolyte interphase (SEI) on the anode surface. Here, we show that FEC addition to KIBs containing hard carbon anodes results in a dramatic decrease in capacity and cell failure in only two cycles, whereas capacity retention remains high ( $>90 \%$ over 80 cycles at $\mathrm{C} / 10$ for both $\mathrm{KPF}_{6}$ and $\mathrm{KFSI}$ ) for electrolytes that do not contain FEC. Using a combination of ${ }^{19} \mathrm{~F}$ solid-state nuclear magnetic resonance (SSNMR) spectroscopy, X-ray photoelectron spectroscopy (XPS), and electrochemical impedance spectroscopy (EIS), we show that FEC decomposes during galvanostatic cycling to form insoluble $\mathrm{KF}$ and $\mathrm{K}_{2} \mathrm{CO}_{3}$ on the anode surface, which correlate with increased interfacial resistance. Our results strongly suggest KIB performance is sensitive to accumulation of an inorganic SEI, likely due to sluggish K diffusion in these compounds. This mechanism of FEC decomposition was confirmed in two separate electrolyte formulations using $\mathrm{KPF}_{6}$ or KFSI. Interestingly, the salt anions do not decompose themselves, unlike their Li analogues. Insight from these results indicates that electrolyte decomposition pathways and favorable SEI components are significantly different in KIBs and LIBs, suggesting that entirely new approaches to KIB electrolyte engineering are needed.
\end{abstract}

Massive installations of grid-scale electrochemical energy storage will be critical to implement renewable, distributed power generation such as wind and solar on a global scale. The majority of existing electrochemical storage utilizes Liion batteries (LIBs), though as demand for Li rapidly rises due to the electrification of transportation, alternative battery chemistries utilizing earth abundant alkali metals, such a $\mathrm{Na}$ and $\mathrm{K}$, are needed for new deployments on the grid to reduce strain on the Li supply chain. ${ }^{1-8} \mathrm{~K}$-ion batteries (KIBs) are especially favored because $\mathrm{K}$ can intercalate in existing graphite anode technologies, whereas intercalation is not thermodynamically favored for $\mathrm{Na}^{7,9-14}$ Additionally, faster transport of $\mathrm{K}$-ions through organic electrolytes (due to a smaller Stokes radius) ${ }^{15}$ compared to Li-ions can enable KIBs that better meet the high-power demands of the grid. ${ }^{16,17}$

Presently, it is unclear if the decades of research dedicated to improving LIB performance is transferable to KIBs. For example, significant research attention in LIBs has focused on engineering a desirable solid electrolyte interphase (SEI), the heterogeneous layer of organic and inorganic decomposition products on the anode surface. ${ }^{18-23}$ It is well established that the chemical composition and physical properties of the SEI are governed by the choice of electrolyte and significantly alter battery performance.4,5,24-26 Specifically, additives such as fluoroethylene carbonate (FEC) have been consistently shown to improve long-term capacity retention in many LIB electrolytes, likely due to their impact on the composition of the SEI. ${ }^{27-37}$ Recent stud- ies on KIBs, however, have shown that FEC dramatically reduces capacity in both intercalation- and alloy-type anodes, though the mechanism for cell failure is not known. ${ }^{38,39}$ The deviation in performance for KIBs suggests that there may be significant differences in electrolyte decomposition pathways and desirable SEI components in KIBs compared to LIBs.

Here, we present a mechanistic description of FEC decomposition and its impact on the composition of KIB SEIs. The SEI deposited on hard carbon anodes in KIBs was examined using a combination of ${ }^{19} \mathrm{~F}$ solid-state nuclear magnetic resonance (SSNMR) spectroscopy, X-ray photoelectron spectroscopy (XPS), and ${ }^{1} \mathrm{H}$ solution NMR to show that FEC decomposes to form $\mathrm{KF}$ and $\mathrm{K}_{2} \mathrm{CO}_{3}$ as well as soluble vinylene carbonate (VC). This result is independent of the choice of $\mathrm{K}$ salt, as cells assembled with $\mathrm{KPF}_{6}$ or KFSI alone show zero or minimal $\mathrm{KF}, \mathrm{K}_{2} \mathrm{CO}_{3}$, and $\mathrm{VC}$ formation. The correlation between these SEI components and poor capacity retention deviates from results in the LIB literature showing that FEC-induced formation of $\mathrm{LiF}$ and polymeric $\mathrm{VC}$ species improves battery performance. ${ }^{37}$ Increases in the interfacial resistance of FEC-containing cells determined via electrochemical impedance spectroscopy (EIS) suggest that $\mathrm{KF}$ and $\mathrm{K}_{2} \mathrm{CO}_{3}$ are poor ionic conductors and that the buildup of an inorganic SEI is unfavorable for KIB performance.

\section{EXPERIMENTAL SECTION}


Materials and Methods. Potassium metal (chunks in mineral oil, 98\% trace metals basis), potassium hexafluorophosphate $\left(\mathrm{KPF}_{6},>99.5 \%\right)$, ethylene carbonate $(\mathrm{EC},>99 \%$, $<10$ ppm $\mathrm{H}_{2} \mathrm{O}$ ), propylene carbonate ( $\mathrm{PC}$, anhydrous, > 99\%), fluoroethylene carbonate (FEC, anhydrous, > 99\%), dimethyl carbonate (DMC, anhydrous, > 99\%), hexanes (anhydrous, > 99\%), and sodium carboxymethyl cellulose (CMC) were purchased from Sigma Aldrich. Potassium bis(fluorosulfonylimide) (KFSI, $>99 \%$, battery grade) was purchased from Synthonix, Inc. Commercially available hard carbon (HC) was provided by Kuraray Co., LTD. Carbon Super P was purchased from MTI Corporation. Deuterated dimethyl sulfoxide (DMSO- $d_{6}, 99.9 \%$ ) was purchased from Cambridge Isotope Laboratories and dried with molecular sieves for at least $24 \mathrm{~h}$. Prior to use, $\mathrm{KPF}_{6}$ was dried in vacuo overnight at $100{ }^{\circ} \mathrm{C}$ before bringing into an Ar-filled glovebox $\left(\mathrm{O}_{2}<0.1 \mathrm{ppm}, \mathrm{H}_{2} \mathrm{O}<0.5 \mathrm{ppm}\right)$. KFSI was dried in vacuo overnight at room temperature (elevated temperatures were not used due to the low melting point of KFSI (102 $\left.{ }^{\circ} \mathrm{C}\right)$ ). EC, PC, and FEC were stored with molecular sieves for at least $24 \mathrm{~h}$ to remove residual water and achieve Karl Fischer titration readings $<10 \mathrm{ppm} \mathrm{H}_{2} \mathrm{O}$ (see Electrolyte Formulations section for more details). All other materials were used as received.

Caution must be taken when handling $K$ metal, as it is extremely reactive and flammable. K metal should only be handled and stored under mineral oil in inert gas in a glovebox. $K$ metal will develop blue/black oxide layers even while stored in mineral oil in $<0.1$ ppm $\mathrm{O}_{2}$ conditions. If $\mathrm{K}$ metal has a yellow/gold hue, it may indicate formation of $\mathrm{KO}_{2}$ superoxide and is possibly explosive.

Electrode Fabrication. Hard carbon electrode films were made by mixing an 8:1:1 mass ratio of hard carbon:carbon Super P:CMC binder. The hard carbon and carbon Super $\mathrm{P}$ were first mixed in a stainless-steel ball mill (SPEX 8000M Mixer/Mill) under Ar for 30 minutes. In a mortar and pestle, water was added dropwise ( $\sim 10$ drops per $100 \mathrm{mg}$ of dry mixture) to the CMC. The carbon mixture was then added from the ball mill and mixed until a slurry was formed. The slurry was cast onto a $\mathrm{Cu}$ current collector ( $6 \mu \mathrm{m}$ thick, MTI) using a $150 \mu \mathrm{m}$ doctor blade and dried at $100{ }^{\circ} \mathrm{C}$ under vacuum overnight. The dried film was punched into $12.7 \mathrm{~mm}$ diameter disks to use in cell assembly. Typical mass loadings of active material per anode were 3-6mg $\mathrm{cm}^{-2}$. These electrodes were used for all electrochemical testing and NMR/XPS characterization.

Electrolyte Formulations. EC and PC were mixed in equal parts by volume and stored with approximately half the volume of molecular sieves for $>24$ hours to dry (performance was found to be strongly dependent on water content, with consistent cycling occurring below 10 ppm $\mathrm{H}_{2} \mathrm{O}$, confirmed by Karl Fischer titration). Four electrolytes of interest were formulated: $0.8 \mathrm{M} \mathrm{KPF}_{6}$ with (denoted $\mathrm{KPF}_{6}+$ FEC) and without (denoted $\mathrm{KPF}_{6}$ ) $5 \mathrm{wt} \% \mathrm{FEC}$ additive and 0.8 M KFSI with (denoted KFSI + FEC) and without (denoted KFSI) $5 \mathrm{wt} \%$ FEC additive. Before mixing the electrolyte, the EC/PC and FEC were filtered using a PTFE filter attached to a syringe to remove residue from the molecular sieves.

Electrochemical Cycling. 2032-type coin cell casings were used to assemble K/hard carbon half cells with $15 \mathrm{~mm}$ diameter glass microfiber separators (purchased from GE
Life Sciences and dried overnight at $60^{\circ} \mathrm{C}$ ). To assemble $\mathrm{K}$ half cells, $\mathrm{K}$ metal was first treated by rinsing thoroughly in hexanes to remove all mineral oil, then the external oxide layer was scraped off with a razor blade in an Ar-filled glovebox. Small pieces of potassium were then placed in a bag coated with hexanes and rolled into thin sheets $(\sim 0.25$ mm thick) using a cylindrical weight. The $\mathrm{K}$ sheet was then removed from the bag and, after waiting for the hexanes to evaporate, stamped into $12.7 \mathrm{~mm}$ diameter disks. Unused $\mathrm{K}$ metal was returned to mineral oil and used within a week of removing the original oxide layer (to avoid new oxide buildup). Coin cells were saturated with 10-12 drops of electrolyte. Galvanostatic cycling experiments were performed with an initial C/50 SEI formation cycle, followed by 10 cycles at $\mathrm{C} / 10$ (where $\mathrm{nC}$ refers to full theoretical discharge in $1 / \mathrm{n}$ hours). C-rates were calculated from the theoretical capacity of hard carbon for the formation of $\mathrm{KC}_{8}$ $\left(279 \mathrm{mAh} \mathrm{g}^{-1}\right)$. Cells were discharged to $0.05 \mathrm{~V} \mathrm{vs} \mathrm{K}^{+} / \mathrm{K}$ and charged to $1.5 \mathrm{~V}$ vs. $\mathrm{K}^{+} / \mathrm{K}$.

Electrode Extraction. For NMR analysis, the hard carbon electrode was removed from the cycled coin cell and dried, unwashed, in an evacuated glovebox antechamber overnight. For XPS analysis, the electrodes were removed and triple rinsed ( $30 \mathrm{~s}$ each rinse) in DMC to remove residual salts and prevent charging in the XPS. They were then dried overnight in an evacuated antechamber to remove residual solvent and prevent off-gassing in the evacuated XPS chamber. Extraction was performed in an Ar-filled glove box within, at most, $12 \mathrm{~h}$ after cells completed cycling.

Solution NMR Measurements. To prepare samples for solution NMR, cycled cells were disassembled inside an Arfilled glovebox. The separator was removed and dipped in 1 $\mathrm{mL}$ of the dried EC/PC solvent used for electrolyte formulation for $30 \mathrm{~s}$ to dissolve soluble decomposition products. The separator was then removed, and the solution was filtered and added to an air-tight J-Young NMR tube. A glass capillary tube containing anhydrous DMSO- $d_{6}$ and sealed with Teflon tape was added to the J-Young for shimming and referencing. DMSO- $d_{6}$ was isolated from the electrolyte solution because DMSO- $d_{6}$ vigorously reacted with K-containing electrolyte (noted by bubble generation upon dipping the separator in DMSO-d6). Pristine electrolytes were formulated and directly added to a J-Young NMR tube with a DMSO- $d_{6}$ capillary. ${ }^{1} \mathrm{H},{ }^{19} \mathrm{~F}$, and ${ }^{31} \mathrm{P}$ solution NMR experiments were performed at room temperature on a Bruker Avance III 400 spectrometer equipped with a triple resonance broadband observe (TBO) probehead. One-dimensional (1D) ${ }^{1} \mathrm{H}\left(30^{\circ}\right.$ single pulse, 1 s recycle delay, 64 scans, internally referenced to DMSO at $2.5 \mathrm{ppm}),{ }^{19} \mathrm{~F}\left(30^{\circ}\right.$ single pulse, 2 s recycle delay, 32 scans, internally referenced to $\mathrm{PF}_{6}{ }^{-}$at $-72.4 \mathrm{ppm}^{-}$or $\mathrm{FSI}^{-}$at $52 \mathrm{ppm}$ ) were collected on all pristine and cycled electrolytes. 1D ${ }^{31} \mathrm{P}\left(30^{\circ}\right.$ single pulse with WALTZ-16 ${ }^{1} \mathrm{H}$ decoupling, $1 \mathrm{~s}$ recycle delay, 128 scans, internally referenced to $\mathrm{PF}_{6}{ }^{-}$at $-146 \mathrm{ppm}$ ) NMR spectra were recorded on $\mathrm{KPF}_{6}$-containing electrolytes.

Solid-state NMR Measurements. SSNMR experiments were performed at room temperature on a Bruker Avance NEO $600 \mathrm{MHz}$ spectrometer equipped with a $1.6 \mathrm{~mm}$ HFXY magic-angle spinning (MAS) Phoenix NMR probehead. After cycling, hard carbon electrodes were extracted as described above and scraped off the $\mathrm{Cu}$ current collector. Two to three 
electrodes were ground together in a mortar and pestle and packed into $1.6 \mathrm{~mm}$ o.d. $\mathrm{ZrO}_{2}$ rotors in an Ar-filled glovebox. All rotors were spun at $25 \mathrm{kHz}$ MAS frequency. All experiments were externally referenced to the ${ }^{1} \mathrm{H}$ NMR resonance of adamantane at $1.85 \mathrm{ppm} .{ }^{1} \mathrm{H},{ }^{13} \mathrm{C}$, and ${ }^{19} \mathrm{~F}$ spectra were collected using a rotor-synchronized Hahn echo $\left(90^{\circ}-\tau-180^{\circ}-\right.$ $\tau$-acquire, where $\tau=1$ rotor period). ${ }^{1} \mathrm{H}$ spectra were acquired in 64 scans with a $10 \mathrm{~s}$ recycle delay. ${ }^{19} \mathrm{~F}$ spectra were acquired in 2048 scans with a recycle delay of $10 \mathrm{~s} .{ }^{13} \mathrm{C}$ spectra were collected with between $44 \mathrm{k}$ and $72 \mathrm{k}$ scans with a recycle delay of $1 \mathrm{~s}$.

XPS measurements. Hard carbon electrodes extracted from cycled cells were washed and dried as described above. The samples were then mounted on XPS stubs inside of the glovebox using carbon tape. Samples were transferred from the glovebox to the XPS in an airtight centrifuge tube, with air contact estimated at $<10 \mathrm{~s}$ per sample during loading. Spectra were collected using a PHI 5600 XPS system with a hemispherical analyzer and an Al X-ray source with XPS base chamber pressure $<1.0 \times 10^{-8}$ Torr. XPS Peak 41 software was used to fit spectra. The adventitious carbon peak in the $\mathrm{C} 1 \mathrm{~s}$ spectrum was referenced to $284.8 \mathrm{eV}$. All peaks were fit using a Shirley baseline correction with two constraints: i) the Gaussian:Lorentzian ratio was the same for all peaks in a given orbital, and ii) the fwhm was the same for all peaks in a given orbital and constrained to $<1.7$ $\mathrm{eV}$.

EIS measurements. Potentiostatic EIS measurements were performed after cells completed cycling on a Biologic SP-150 potentiostat using a frequency range of $1 \mathrm{MHz}$ to 0.1 $\mathrm{Hz}$ with a voltage perturbation of $10 \mathrm{mV}$. Fitting was performed using Zahner Analysis software (fit details can be found in the Supporting Information (SI)).

\section{RESULTS}

Electrochemical performance. The specific discharge capacities for cells cycled in $\mathrm{KPF}_{6}$ and KFSI electrolyte salts, with and without FEC, are shown as a function of cycle number in Figure 1. Slow electrochemical cycling was used to generate a SEI in the first cycle. In the first C/50 formation cycle, all cells had specific capacities near the theoretical capacity of $279 \mathrm{mAh} \mathrm{g}^{-1}\left(265,330,324\right.$, and $257 \mathrm{mAh} \mathrm{g}^{-1}$ for $\mathrm{KPF}_{6}, \mathrm{KPF}_{6}+\mathrm{FEC}, \mathrm{KFSI}$, and $\mathrm{KFSI}+\mathrm{FEC}$, respectively). In subsequent cycles, a cycling rate of $\mathrm{C} / 10$ is used, and specific capacities of the $\mathrm{KPF}_{6}$ and KFSI cells drop to $45 \%$ and $52 \%$ of the initial capacity, respectively, which is reasonable given the change in C-rate. ${ }^{25,40}$ Specific capacity decreases significantly more in $\mathrm{KPF}_{6}+\mathrm{FEC}$ and $\mathrm{KFSI}+\mathrm{FEC}$ cells, to just $7 \%$ and $8 \%$ of the initial capacity. By the third cycle, specific capacities for all cells have plateaued, and by the eleventh, cycle specific capacities for $\mathrm{KPF}_{6}, \mathrm{KPF}_{6}+\mathrm{FEC}, \mathrm{KFSI}$, and KFSI + FEC are $106 \mathrm{mAh} \mathrm{g}^{-1}, 3 \mathrm{mAh} \mathrm{g}^{-1}, 169 \mathrm{mAh} \mathrm{g}^{-1}$, and $3 \mathrm{mAh}$ $\mathrm{g}^{-1}$, respectively. While KFSI electrolytes exhibit higher reversible capacities compared to $\mathrm{KPF}_{6}$ in cells without FEC, these improvements are eliminated upon FEC addition. The same pattern of rapid capacity decline due to FEC addition is seen in several other solvent combinations (i.e., PC and EC/DMC, Figure S1). Although KIBs containing FEC show extremely poor capacity retention, electrolytes without FEC show stable cycling behavior. For $\mathrm{KPF}_{6}$, reversible capacity stabilized after 15 cycles and $99 \%$ is retained by cycle 80 ;

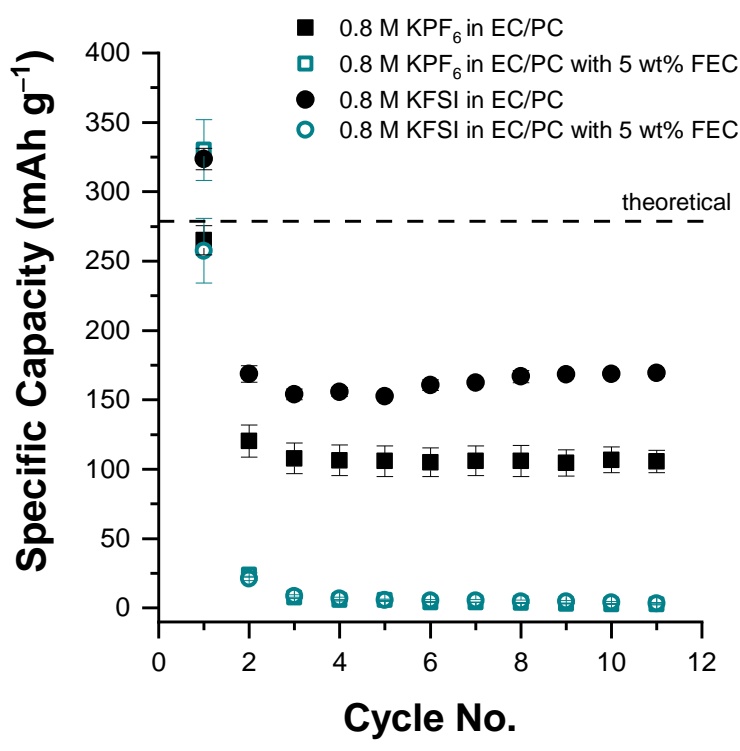

Figure 1. Specific capacity of HC/K half cells cycled at $\mathrm{C} / 10$ (with one formation cycle at C/50). Cells were formulated with either $\mathrm{KPF}_{6}$ (squares) or KFSI (circles) salts in EC/PC, and with (teal, open symbols) or without (black, solid symbols) 5 wt\% FEC. Plotted capacities are the average of three cells.

for KFSI, $89 \%$ of reversible capacity is retained in the first 80 cycles; Figure S2), strongly suggesting that FEC leads to unfavorable changes in the composition of the SEI.

Solution NMR characterization of soluble electrolyte decomposition products. To characterize soluble decomposition products, ${ }^{1} \mathrm{H}$ NMR spectra were obtained for cells cycled in all electrolyte formulations (Figure 2; full spectra are shown in Figure S6). All spectra are dominated by peaks corresponding to the electrolyte solvents. EC appears as a singlet (s) at $4.15 \mathrm{ppm}$. PC exhibits four unique ${ }^{1} \mathrm{H}$ shifts: the doublet (d) at $1.09 \mathrm{ppm}\left({ }^{3} \mathrm{~J}_{\mathrm{H}-\mathrm{H}}=6.3 \mathrm{~Hz}\right)$ corresponds to the methyl protons; the two doublet of doublets $\left(\mathrm{dd},{ }^{2} J_{\mathrm{H}-\mathrm{H}}=8.9\right.$ $\mathrm{Hz},{ }^{3} \mathrm{~J}-\mathrm{H}=7.2 \mathrm{~Hz}$ ) centered at 3.72 and $4.25 \mathrm{ppm}$ are assigned to the two inequivalent protons in the methylene group; the multiplet (m) at $4.57 \mathrm{ppm}$ corresponds to the methine proton on the five-membered ring. In the samples with FEC additives, peaks corresponding to FEC are observed as a doublet of doublet of doublets (ddd) at $6.11 \mathrm{ppm}$ $\left({ }^{3} J_{\mathrm{H}-\mathrm{H}}=1.0 \mathrm{~Hz},{ }^{2} J_{\mathrm{H}-\mathrm{H}}=4.2 \mathrm{~Hz},{ }^{2} J_{\mathrm{F}-\mathrm{H}}=64.5 \mathrm{~Hz}\right)$ for the methine proton and another ddd at $4.76 \mathrm{ppm}$ that is assigned to one of the two inequivalent protons in the methylene group. The other proton is obscured by the ${ }^{1} \mathrm{H}$ resonances from the PC solvent. Figures S3 and S4 show the corresponding ddd assigned to FEC at $-122 \mathrm{ppm}$ in ${ }^{19} \mathrm{~F}$ NMR. Additionally, a singlet peak at $7.05 \mathrm{ppm}$ is assigned to vinylene carbonate, ${ }^{37} \mathrm{a}$ known product of FEC reduction. The slight difference in the ${ }^{1} \mathrm{H}$ shift that we observe for $\mathrm{VC}$ compared to previous literature $(7.05 \mathrm{ppm} \text { vs } 7.77 \mathrm{ppm})^{31}$ is likely due to solvent effects, ${ }^{41,42}$ since we do not mix our spent electrolyte with DMSO- $d_{6}$ to avoid further decomposition reactions and rather dissolve in the parent EC/PC solvent. Regardless of salt choice, FEC appears to undergo a similar reduction mechanism in KIBs as in LIBs to generate soluble, unsaturated organic species. With the exception of a few small peaks from 

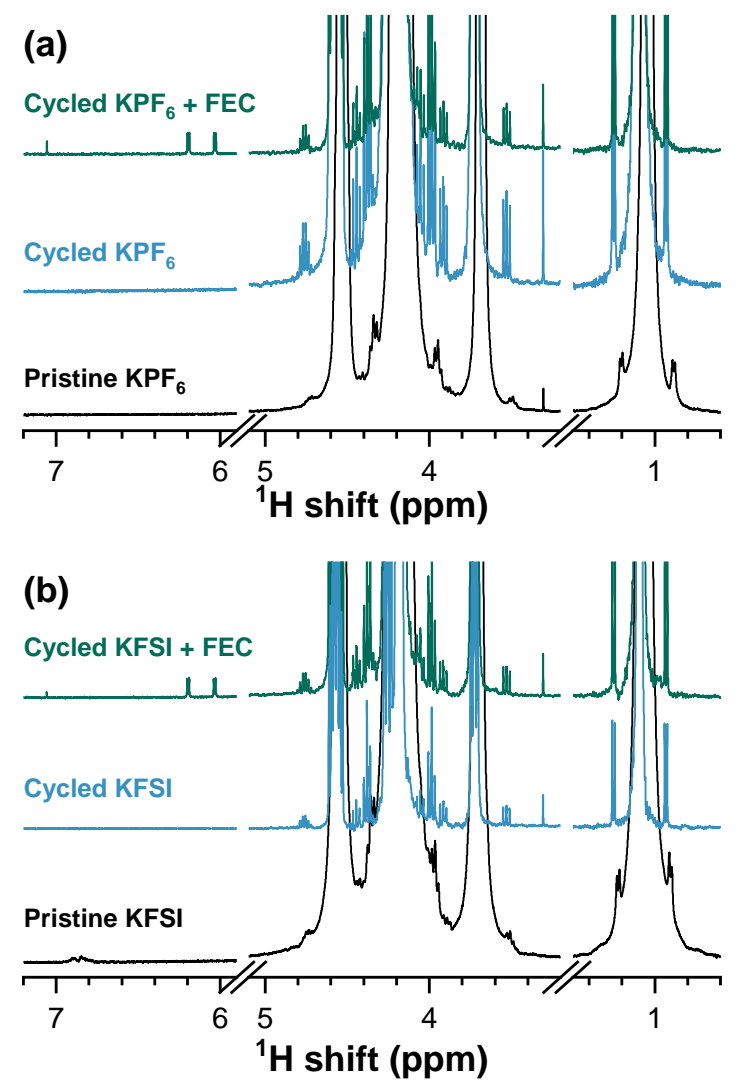

Figure 2. Ex situ ${ }^{1} \mathrm{H}$ solution NMR of pristine (black spectra) and cycled electrolytes (light blue and teal spectra, 11 galvanostatic cycles in $\mathrm{K} / \mathrm{HC}$ half cells; first cycle at $\mathrm{C} / 50$, subsequent cycles at $\mathrm{C} / 10$ ) in (a) $0.8 \mathrm{M}$ $\mathrm{KPF}_{6}$ or (b) KFSI in EC/PC both with (top, teal) or without (middle, light blue) 5\% FEC.

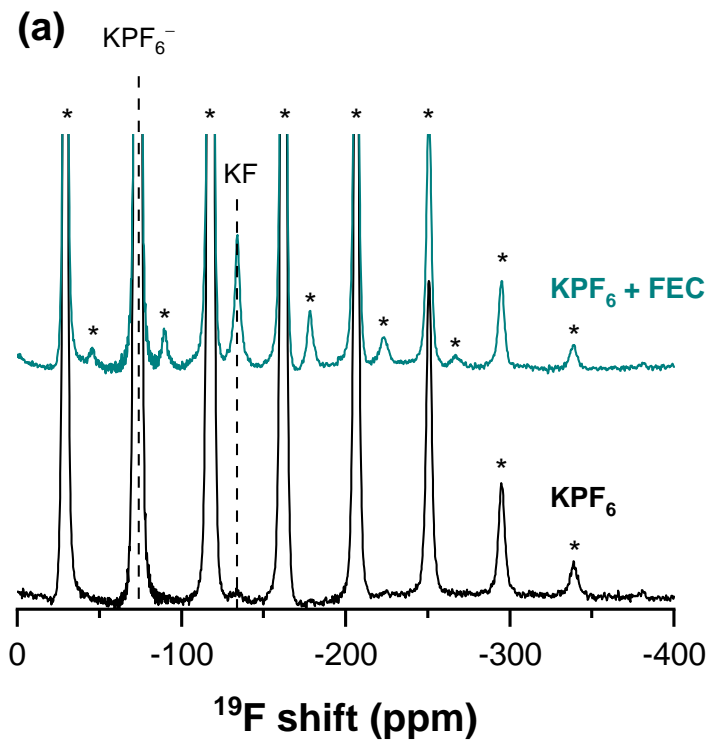

trace solvent impurities between $3.2 \mathrm{ppm}$ and $1.5 \mathrm{ppm}$ (Figure S6), no other soluble decomposition products are detected in ${ }^{1} \mathrm{H}$ (Figure 2, light blue spectra), ${ }^{19} \mathrm{~F}$ (Figures $\mathrm{S} 3$ and S4), or ${ }^{31}$ P NMR (Figure S5). This differs from LIBs, where numerous short-chain organic Li salts (e.g., Li ethylene decarbonate (LEDC), Li ethylmethyl carbonate (LEMC), 2,5-dioxahexanedioic acid methyl ester (DMDOHC), 2-methoxyethyl methyl carbonate (MEMC), Li propyl dimethylcarbonate (LPDC)) and organic small molecules (e.g., propylene glycol, formic acid, and acetic acid) have been detected in solution-state NMR from EC/PC decomposition..$^{21,37,43}$ The lack of new peaks in ${ }^{1} \mathrm{H}$ solution NMR suggests either that minimal solvent decomposition occurs and/or that the products are insoluble and accumulate on the surface of the electrodes. The lack of organic decomposition is consistent with the lower reduction potential of carbonate solvents measured in $\mathrm{K}$ electrolytes, ${ }^{44}$ as well as DFT calculations that show $\mathrm{K}^{+}$coordination with solvent molecules hinders charge transfer between the electrode surface and solvent, mitigating solvent reduction. ${ }^{45}$

Solid-state NMR characterization of the SEI on hard carbon anodes after electrochemical cycling in KIBs. To probe changes in SEI composition due to FEC additives on the electrode surface, ex situ ${ }^{1} \mathrm{H},{ }^{13} \mathrm{C}$, and ${ }^{19} \mathrm{~F}$ SSNMR spectra were collected for $\mathrm{HC}$ electrodes after eleven galvanostatic cycles. ${ }^{19} \mathrm{~F}$ SSNMR spectra show that FEC causes significant changes to the inorganic components of the SEI on HC (Figure 3). All spectra shown in Figure 3 have been normalized with respect to the mass of the sample and the number of scans to allow for direct quantitative comparisons. Figure 3a shows spectra collected for $\mathrm{HC}$ cycled in $\mathrm{KPF}_{6}$ (black, bottom) and $\mathrm{KPF}_{6}+\mathrm{FEC}$ (teal, top). The doublet at $-73 \mathrm{ppm}\left(\mathrm{JF}_{-}\right.$ $\mathrm{p}=672 \mathrm{~Hz}$, full non-truncated ${ }^{19} \mathrm{~F}$ spectra shown in Figure $\mathrm{S7}$ ) is assigned to residual $\mathrm{PF}_{6}$ - from the electrolyte salt. The peak at $-134 \mathrm{ppm}$ matches that of bulk KF. ${ }^{46}$ The KF peak is significantly larger in the sample with FEC, suggesting that

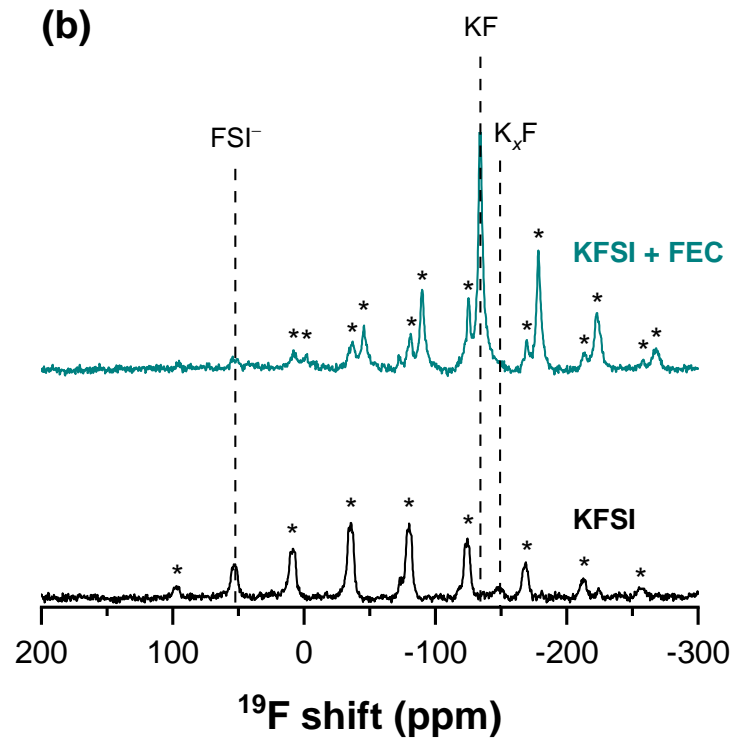

Figure 3. Ex situ ${ }^{19} \mathrm{~F}$ solid-state NMR of hard carbon anodes after 11 galvanostatic cycles (first cycle at $\mathrm{C} / 50$, subsequent cycles at C/10) in $0.8 \mathrm{M} \mathrm{KPF}_{6}$ (a) or KFSI (b) in EC/PC both with (teal spectra) or without (black spectra) 5 wt\% FEC. All samples were spun at $25 \mathrm{kHz}$ MAS frequency. Asterisks denote spinning sidebands. 
it is a major product of FEC reduction and may contribute to rapid capacity fade.

A similar trend is observed in cells containing KFSI-based electrolytes both with and without FEC (Figure 3b). With $\mathrm{KFSI}$ alone (teal, top), no KF is observed, whereas a large $\mathrm{KF}$ peak is present when FEC is added to the electrolyte formulation (black, bottom). The intensity of the KF peaks in all cells made with FEC are similar (as was the amount of FEC initially added), suggesting that the same amount of KF was produced and is independent of salt choice. Again, this suggests that large amounts of $\mathrm{KF}$ in the SEI on $\mathrm{HC}$ are correlated with rapid cell death. In the KFSI electrolyte, we observe a small ${ }^{19} \mathrm{~F}$ resonance at $-144 \mathrm{ppm}$, which is tentatively assigned to a partially potassiated fluoride compound (e.g., $\mathrm{K}_{\mathrm{x}} \mathrm{F}$ ), possibly from $\mathrm{FSI}^{-}$decomposition which may produce different fluorinated products compared to FEC. ${ }^{47}$ The ${ }^{19} \mathrm{~F}$ resonance at $53 \mathrm{ppm}$ is assigned to residual $\mathrm{FSI}^{-}$anions and is observed in all KFSI-containing samples as electrodes are not washed prior to SSNMR analyses. Additionally, a small peak at $-223 \mathrm{ppm}$ is seen in the KFSI sample, and is assigned to the Torlon rotor cap, ${ }^{45}$ which contains small amounts of $\mathrm{F}$.

Extracting information on the SEI from ${ }^{1} \mathrm{H}$ and ${ }^{13} \mathrm{C}$ SSNMR measurements is more challenging than ${ }^{19} \mathrm{~F}$ SSNMR due to the broad signals originating from the HC electrode. For example, broad resonances from the aromatic carbon substrate span from approximately 220 to $-10 \mathrm{ppm}$ in ${ }^{13} \mathrm{C}$ SSNMR, encompassing the entire range where organic decomposition products are expected (Figures S8 and S9). This broad lineshape arises from the amorphous nature of HC, where the material exhibits several unique $C$ sites, leading to a distribution of chemical shift values. $\mathrm{HC}$ also contains delocalized electrons that can couple to nuclei in the material and SEI, causing additional line broadening that worsens chemical shift resolution in ${ }^{1} \mathrm{H}$ SSNMR. As the quantity of active material is likely much greater than that of the surface-bound SEI, ${ }^{1} \mathrm{H}$ and ${ }^{13} \mathrm{C}$ SSNMR spectra are overwhelmed by HC and only the KFSI-containing samples show a discernable ${ }^{13} \mathrm{C}$ resonance at $\sim 68 \mathrm{ppm}$, which is assigned to small amounts of poly(ethylene oxide) (PEO)-type structures that are generated during EC/PC reduction.

XPS characterization of the SEI on hard carbon anodes after electrochemical cycling in KIBs. To corroborate our findings from SSNMR and gain additional insight into compositional changes to the carbon-containing SEI, we examined the surface of cycled $\mathrm{HC}$ anodes with $\mathrm{C} 1 \mathrm{~s}$ and $\mathrm{F}$ 1s XPS (vide supra, ${ }^{1} \mathrm{H}$ and ${ }^{13} \mathrm{C}$ SSNMR (Figures S8 and S9) show a large background signal from the underlying $\mathrm{HC}$ anode and cannot be used to identify organic compounds in the SEI). F 1s XPS spectra (Figure S10) confirm assignments from ${ }^{19} \mathrm{~F}$ SSNMR for all electrolyte formulations. For example, peaks corresponding to $\mathrm{KF}$ at approximately $684.7 \mathrm{eV}$ only appear in samples containing FEC, which is in good agreement with findings from SSNMR. In the KFSI sample, a fluoride-like compound, with a peak that is resolved from $\mathrm{KF}$, is detected at $685.5 \mathrm{eV}$ and assigned to the $\mathrm{K}_{\mathrm{x}} \mathrm{F}$ phase found in ${ }^{19} \mathrm{~F}$ SSNMR. The relative amounts of residual $\mathrm{PF}_{6}$ and $\mathrm{FSI}^{-}$anions detected in F 1s XPS vary from sample to sample, likely due to the washing procedure required to prevent charging in the XPS.

Figure 4 shows $\mathrm{C}$ 1s XPS of HC anodes cycled in all electrolyte formulations. All spectra can be fitted with peaks corresponding to $\mathrm{C}-\mathrm{C}, \mathrm{C}-\mathrm{O}$, and $\mathrm{C}=\mathrm{O}$ bonding environments. The ratio of these three bond environments is similar between all samples except for $\mathrm{KPF}_{6}$ without FEC (Figure 4a). The increased proportion of $\mathrm{C}=0$ bonds is assigned to the
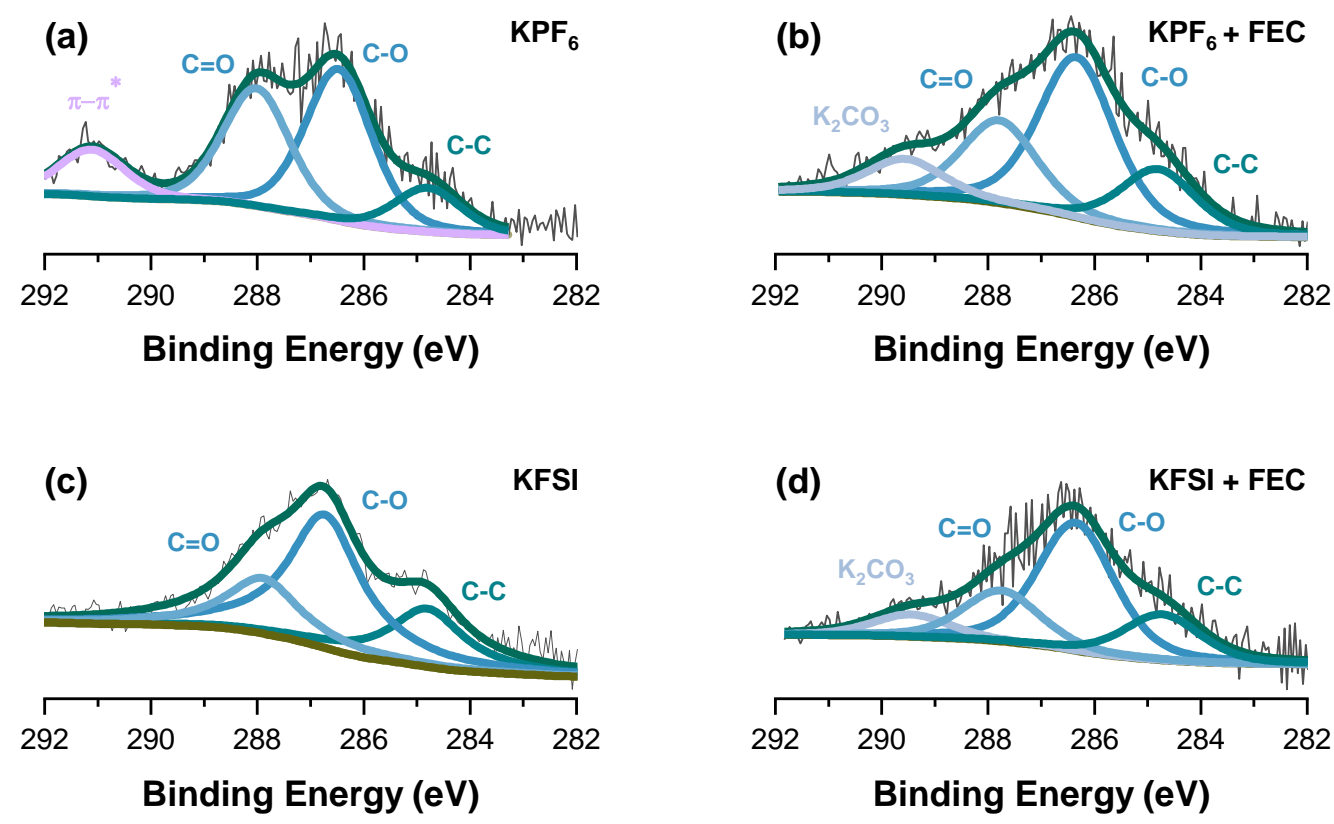

Figure 4. Ex situ XPS of the C 1 s orbital for $\mathrm{HC} / \mathrm{K}$ cells formulated with $0.8 \mathrm{M} \mathrm{KPF}_{6}$ or KFSI in EC/PC with (b, d, respectively) and without (a, c, respectively) 5 wt \% FEC. All cells underwent an initialization cycle at C/50, followed by ten C/10 cycles. 
(a)

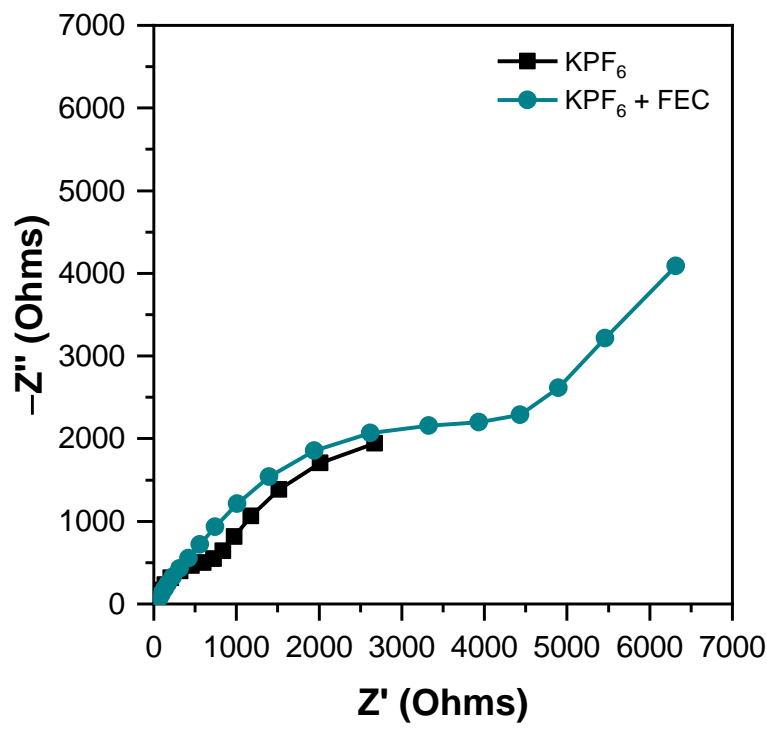

(b)

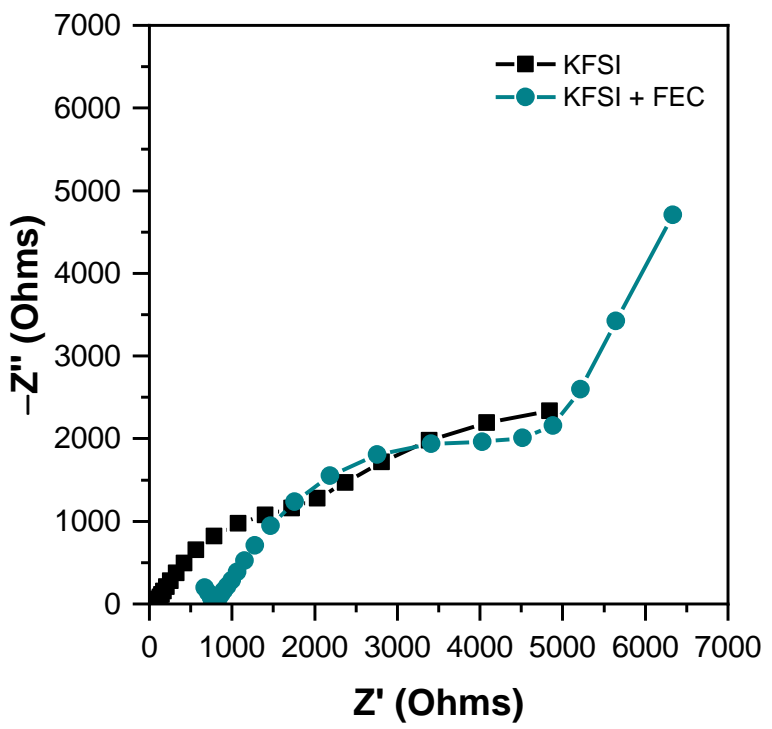

Figure 5. Electrochemical impedance spectra collected after eleven cycles (one cycle at $\mathrm{C} / 50$ and ten at $\mathrm{C} / 10$ ) of $\mathrm{HC} / \mathrm{K}$ half cells in $0.8 \mathrm{M} \mathrm{KPF}_{6}$ (left) or KFSI (right) in EC/PC both with (teal circles) or without (black squares) $5 \mathrm{wt} \% \mathrm{FEC}$.

CMC binder from the underlying electrode. This assignment is supported by the appearance of a C 1 s peak at $291 \mathrm{eV}$ from the $\pi-\pi^{*}$ satellites in hard carbon and suggest that a thinner SEI is generated in $\mathrm{KPF}_{6}$ electrolyte that allows detection of HC. Notably, all HC anodes cycled with FEC in the electrolyte formulation exhibit an additional peak at 289.6 $\mathrm{eV}$, which is assigned to $\mathrm{K}_{2} \mathrm{CO}_{3},{ }^{48}$ independent of electrolyte salt. The presence of $\mathrm{K}_{2} \mathrm{CO}_{3}$ in the SEI of $\mathrm{HC}$ cycled in FECcontaining electrolytes (Figure $4 \mathrm{~b}$ and $4 \mathrm{~d}$ ) agrees with the LIB literature, where $\mathrm{Li}_{2} \mathrm{CO}_{3}$ is a known byproduct from FEC reduction. ${ }^{29}$ The fact that inorganic $\mathrm{K}$ compounds $\left(\mathrm{K}_{2} \mathrm{CO}_{3}\right.$ and KF) are only detected when FEC is present suggests that these compounds may be responsible for the extremely poor capacity retention observed during galvanostatic cycling measurements (Figure 1).

Interfacial resistance increases upon addition of FEC to KIB electrolytes. Electrochemical impedance spectra were collected after eleven cycles for cells cycled in all electrolyte formulations (Figure 5). Regardless of the choice of salt, FEC addition increases interfacial resistance (resistances are listed in Table S1). The first semicircle in the low-frequency region represents impedance of the SEI layer, and the second semicircle in the high-frequency region corresponds to impedance due to $\mathrm{K}$ diffusion (the corresponding resistances are proportional to the diameter of each semicircle). By fitting the data with a standard Randles model (Figures S11 and S12), ${ }^{49}$ we see that the SEI resistance increases with FEC addition for both electrolyte salts from 3.8 to $8.1 \mathrm{kOhm}$ in $\mathrm{KPF}_{6}$ and from 4.5 to 15.5 $\mathrm{kOhm}$ in KFSI (Table S1 lists all values and errors associated with the EIS fits). The results from SSNMR and XPS show that this increase in interfacial resistance coincides with the formation of inorganic salts ( $\mathrm{KF}$ and $\mathrm{K}_{2} \mathrm{CO}_{3}$ ) due to FEC decomposition, suggesting that these compounds are poor ionic conductors and likely hinder $\mathrm{K}^{+}$transport to the active material.

\section{DISCUSSION}

Characterization of the SEI on HC in KIBs with NMR, XPS, and EIS indicates that reduction products from FEC additives that are beneficial for LIBs (e.g., metal fluorides) have a deleterious effect on KIB performance. We propose that the formation of $\mathrm{KF}$ and $\mathrm{K}_{2} \mathrm{CO}_{3}$ from FEC decomposition increases the interfacial resistance of the cell and is responsible for the rapid capacity fade observed in FEC-containing KIBs. Poor ionic conductivity of $\mathrm{KF}$ and $\mathrm{K}_{2} \mathrm{CO}_{3}$ in the SEI on $\mathrm{HC}$ is supported by EIS measurements that show large increases in interfacial resistance when FEC is present in KIBs (therefore coinciding with $\mathrm{KF}$ and $\mathrm{K}_{2} \mathrm{CO}_{3}$ formation), regardless of salt choice. High interfacial resistance values are expected due to sluggish $\mathrm{K}$ diffusion through the inorganic compounds in the SEI, possibly due to the larger size of $\mathrm{K}$ compared to Li. Both $\mathrm{KF}$ and $\mathrm{K}_{2} \mathrm{CO}_{3}$ exhibit lower dielectric constants compared to their Li counterparts; the room temperature dielectric constant of $\mathrm{KF}$ is $39 \%$ lower than that of $\mathrm{LiF}$ (and 8\% higher than NaF). ${ }^{50}$ Lower dielectric constants in binary salts lead to less charge screening and higher activation barriers to ion diffusion. ${ }^{51}$ In recent computational work, Greeley and coworkers suggested that this trend holds for alkali-fluoride salts and predicted that the conductivity of $\mathrm{NaF}$ is ten orders of magnitude lower than LiF. ${ }^{52} \mathrm{As}$ a result, we expect that KIB performance will also suffer with increasing quantities of inorganic salts in the SEI, contrary to LIBs which benefit from LiF formation. ${ }^{53,54}$

Solution and solid-state ${ }^{19} \mathrm{~F}$ NMR measurements also indicate that KIB and LIB electrolytes follow different decomposition pathways altogether. For example, $\mathrm{KPF}_{6}$ and KFSI are not reduced to $\mathrm{KF}$, while their Li analogues readily decompose to form LiF.45,55 In contrast, only KFSI decomposes to form small amounts of a partially potassiated $\mathrm{K}_{\mathrm{x}} \mathrm{F}$ phase that does not decrease capacity retention to the same extent 
as KF. Likewise, $\mathrm{KPF}_{6}$ does not undergo a hydrolysis reaction with trace water in the electrolyte solvent like $\mathrm{LiPF}_{6}, 56,57$ limiting the amount of $\mathrm{KF}, \mathrm{HF}$, and fluorophosphates that are formed in KIBs. Based on the correlation between electrochemical performance (both cycling and EIS) and inorganic compounds in the SEI, we expect that the high stability of KFSI and $\mathrm{KPF}_{6}$ is beneficial to KIB chemistries, and that efforts to passivate the anode should focus on organic protection layers.

Unfortunately, the lower reduction potential observed for $\mathrm{K}^{+}$compared to $\mathrm{Li}^{+}$electrolytes ${ }^{44}$ likely leads to less organic solvent decomposition in KIBs compared to LIBs. Little to no EC/PC reduction products are observed in solution ${ }^{1} \mathrm{H}$ NMR after cycling of KIBs, which is in stark contrast to welldescribed pathways observed in LIB chemistries that produce myriad soluble, organic products (e.g., LEDC, LPDC, LEMC, etc.). Although small quantities of C-O and PEO-type structures are observed in XPS and SSNMR, detection of the underlying HC anode in XPS suggests that the organic SEI is relatively thin and may not protect the electrolyte from decomposition at the electrode surface. Although $\mathrm{K}_{2} \mathrm{CO}_{3}$ can arise from EC/PC reduction, the fact that it is only observed in the presence of FEC-containing electrolytes indicates that $\mathrm{K}_{2} \mathrm{CO}_{3}$ is primarily generated from $\mathrm{FEC}$ reduction pathways. A potential decomposition mechanism for FEC in KIBs that produces $\mathrm{KF}, \mathrm{K}_{2} \mathrm{CO}_{3}$, and $\mathrm{VC}$ observed in NMR and XPS is shown in Scheme 1. In this reaction, $\mathrm{K}^{+}$initially reduces $\mathrm{FEC}$ to form $\mathrm{KF}$ and an EC radical. Further reductions of the EC radical result in formation of soluble VC structures observed in solution NMR and insoluble $\mathrm{KF}$ and $\mathrm{K}_{2} \mathrm{CO}_{3}$ compounds observed in SEI characterizations. ${ }^{29,31,37}$

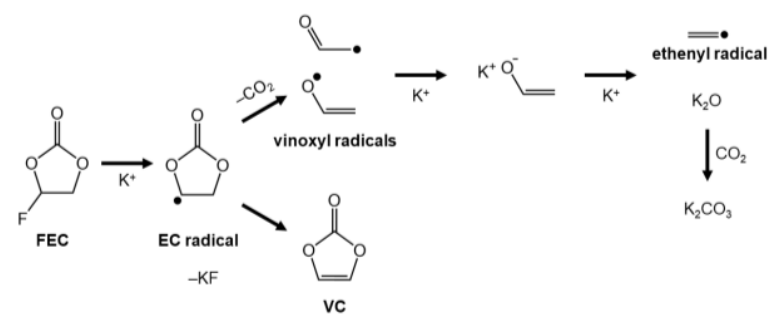

Scheme 1. Reaction scheme showing reduction and decomposition of FEC in KIBs to ultimately form VC, KF, and $\mathrm{K}_{2} \mathrm{CO}_{3}$.

\section{CONCLUSION}

Mechanistic insight from NMR, XPS, and EIS strongly suggest that electrolyte engineering principles from $\mathrm{Li}$ and $\mathrm{Na}-$ ion batteries are not simply transferrable to $\mathrm{K}$-ion systems due to differences in decomposition mechanisms and the physical properties of K-based SEI components. In general, it appears that the buildup of inorganic salts in the SEI ruins KIB performance, likely due to slow $\mathrm{K}$ diffusion at the electrode/electrolyte interface and increased interfacial resistance. When engineering KIB electrolytes, K salts and additives must be carefully selected to avoid decomposition to $\mathrm{KF}$ and other insoluble salts, though the demonstrated stability of K salts makes this choice relatively straightforward. Since fluorinated additives commonly used in LIBs will likely be reduced to form $\mathrm{KF}$, this study suggests that entirely new additives must be investigated for use in KIBs. Alternatively, the formation of stable, ionically conductive SEIs may be possible through engineering of an artificial SEI prior to electrochemical cycling.

\section{ASSOCIATED CONTENT}

Supporting Information. This material is available free of charge on the ACS Publications website at DOI:

Additional electrochemical cycling data, solution NMR, solid-state NMR, XPS, and EIS data, including Figures S1-S12 and Table S1

\section{AUTHOR INFORMATION}

\section{Corresponding Author \\ *lem2221@columbia.edu}

\section{Author Contributions}

A.W.E. assembled all cells and carried out all characterization. R.M. assisted with collection and analysis of XPS data. A.W.E. and L.E.M. conceived of and designed the study. The manuscript was written through contributions of all authors. All authors have given approval to the final version of the manuscript.

\section{Funding Sources}

NSF DMR-2116728.

\section{Notes}

The authors declare no competing financial interest. Raw NMR data may be found on the Open Science Framework (OSF) at https://osf.io/rp6sv/. All other data are available from the authors upon request.

\section{ACKNOWLEDGMENT}

This work was supported by the National Science Foundation (DMR-2116728). A.W.E. and R.M. are both supported by the U.S. Department of Defense through the National Defense Science \& Engineering Graduate Fellowship (NDSEG) program.

\section{REFERENCES}

1. Speirs, J., Contestabile, M., Houari, Y. \& Gross, R. The future of lithium availability for electric vehicle batteries. Renew. Sustain. Energy Rev. 35, 183-193 (2014).

2. Greim, P., Solomon, A. A. \& Breyer, C. Assessment of lithium criticality in the global energy transition and addressing policy gaps in transportation. Nat. Commun. 11, 1-11 (2020).

3. Martin, G., Rentsch, L., Höck, M. \& Bertau, M. Lithium market research - global supply, future demand and price development. Energy Storage Mater. 6, 171-179 (2017).

4. Dhir, S., Wheeler, S., Capone, I. \& Pasta, M. Outlook on K-Ion Batteries. Chem 6, 2442-2460 (2020). 
5. Hosaka, T., Kubota, K., Hameed, A. S. \& Komaba, S. Research Development on K-Ion Batteries. Chem. Rev. 120, 6358-6466 (2020).

6. Eftekhari, A., Jian, Z. \& Ji, X. Potassium Secondary Batteries. ACS Appl. Mater. Interfaces 9, 44044419 (2017).

7. Vaalma, C., Buchholz, D., Weil, M. \& Passerini, S. A cost and resource analysis of sodium-ion batteries. Nat. Rev. Mater. 3, (2018).

8. Ma, Y. et al. An Alternative Charge-Storage Mechanism for High-Performance Sodium-Ion and Potassium-Ion Anodes. ACS Energy Lett. 6, 915-924 (2021).

9. Adams, R. A., Varma, A. \& Pol, V. G. Temperature dependent electrochemical performance of graphite anodes for K-ion and Li-ion batteries. J. Power Sources 410-411, 124-131 (2019).

10. Komaba, S., Hasegawa, T., Dahbi, M. \& Kubota, K. Potassium intercalation into graphite to realize high-voltage/high-power potassium-ion batteries and potassium-ion capacitors. Electrochem. commun. 60, 172-175 (2015).

11. Wang, L. et al. Graphite as a potassium ion battery anode in carbonate-based electrolyte and etherbased electrolyte. J. Power Sources 409, 24-30 (2019).

12. Zhao, J., Zou, X., Zhu, Y., Xu, Y. \& Wang, C. Electrochemical Intercalation of Potassium into Graphite. Adv. Funct. Mater. 26, 8103-8110 (2016).

13. Jian, Z., Luo, W. \& Ji, X. Carbon Electrodes for KIon Batteries. J. Am. Chem. Soc. 137, 1156611569 (2015).

14. Dou, X. et al. Hard carbons for sodium-ion batteries: Structure, analysis, sustainability, and electrochemistry. Mater. Today 23, 87-104 (2019).

15. Matsuda, Y., Nakashima, H., Morita, M. \& Takasu, Y. Behavior of Some Ions in Mixed Organic Electrolytes of High Energy Density Batteries. J. Electrochem. Soc. 128, 2552-2556 (1981).

16. Ji, X. A paradigm of storage batteries. Energy Environ. Sci. 12, 3203-3224 (2019).

17. Davies, D. M. et al. Combined economic and technological evaluation of battery energy storage for grid applications. Nat. Energy 4, 4250 (2019).

18. Peled, E. \& Menkin, S. Review-SEI: Past, Present and Future. J. Electrochem. Soc. 164, A1703A1719 (2017).

19. Winter, M., Barnett, B. \& Xu, K. Before Li Ion Batteries. Chem. Rev. 118, 11433-11456 (2018).

20. $\mathrm{Xu}, \mathrm{K}$. Nonaqueous liquid electrolytes for lithiumbased rechargeable batteries. Chem. Rev. 104, 4303-4417 (2004).
21. Wang, A., Kadam, S., Li, H., Shi, S. \& Qi, Y. Review on modeling of the anode solid electrolyte interphase (SEI) for lithium-ion batteries. npj Comput. Mater. 4, (2018).

22. Heiskanen, S. K., Kim, J. \& Lucht, B. L. Generation and Evolution of the Solid Electrolyte Interphase of Lithium-Ion Batteries. Joule 3, 2322-2333 (2019).

23. An, S. J. et al. The state of understanding of the lithium-ion-battery graphite solid electrolyte interphase (SEI) and its relationship to formation cycling. Carbon N. Y. 105, 52-76 (2016).

24. Hui, J. et al. Achieving Fast and Efficient $\mathrm{K}+$ Intercalation on Ultrathin Graphene Electrodes Modified by a $\mathrm{Li}+$ Based Solid-Electrolyte Interphase. J. Am. Chem. Soc 140, 45 (2018).

25. Hosaka, T., Matsuyama, T., Kubota, K., Yasuno, S. \& Komaba, S. Development of KPF6/KFSA Binary-Salt Solutions for Long-Life and HighVoltage K-Ion Batteries. ACS Appl. Mater. Interfaces 12, 34873-34881 (2020).

26. Leskes, M. et al. Surface-Sensitive NMR Detection of the Solid Electrolyte Interphase Layer on Reduced Graphene Oxide. 8, 1078-1085 (2017).

27. Profatilova, I. A., Kim, S. S. \& Choi, N. S. Enhanced thermal properties of the solid electrolyte interphase formed on graphite in an electrolyte with fluoroethylene carbonate. Electrochim. Acta 54, 4445-4450 (2009).

28. $\mathrm{Xu}, \mathrm{C}$. et al. Improved performance of the silicon anode for li-ion batteries: Understanding the surface modification mechanism of fluoroethylene carbonate as an effective electrolyte additive. Chem. Mater. 27, 2591-2599 (2015).

29. Michan, A. L. et al. Fluoroethylene Carbonate and Vinylene Carbonate Reduction: Understanding Lithium-Ion Battery Electrolyte Additives and Solid Electrolyte Interphase Formation. (2016). doi:10.1021/acs.chemmater.6b02282

30. Markevich, E., Salitra, G. \& Aurbach, D. Fluoroethylene Carbonate as an Important Component for the Formation of an Effective Solid Electrolyte Interphase on Anodes and Cathodes for Advanced Li-Ion Batteries. ACS Energy Lett. 2, 1337-1345 (2017).

31. Jin, Y. et al. Identifying the Structural Basis for the Increased Stability of the Solid Electrolyte Interphase Formed on Silicon with the Additive Fluoroethylene Carbonate. J. Am. Chem. Soc. 139, 14992-15004 (2017).

32. Etacheri, V. et al. Effect of fluoroethylene carbonate (FEC) on the performance and surface chemistry of Si-nanowire li-ion battery anodes. Langmuir 28, 965-976 (2012).

33. Mogi, R. et al. Effects of Some Organic Additives 
on Lithium Deposition in Propylene Carbonate. J. Electrochem. Soc. 149, A1578 (2002).

34. Nakai, H., Kubota, T., Kita, A. \& Kawashima, A. Investigation of the Solid Electrolyte Interphase Formed by Fluoroethylene Carbonate on $\mathrm{Si}$ Electrodes. J. Electrochem. Soc. 158, A798 (2011).

35. Thenuwara, A. C. et al. Efficient LowTemperature Cycling of Lithium Metal Anodes by Tailoring the Solid-Electrolyte Interphase. ACS Energy Lett. 5, 2411-2420 (2020).

36. Zhang, X. Q., Cheng, X. B., Chen, X., Yan, C. \& Zhang, Q. Fluoroethylene Carbonate Additives to Render Uniform Li Deposits in Lithium Metal Batteries. Adv. Funct. Mater. 27, 1-8 (2017).

37. Jin, Y. et al. Understanding Fluoroethylene Carbonate and Vinylene Carbonate Based Electrolytes for $\mathrm{Si}$ Anodes in Lithium Ion Batteries with NMR Spectroscopy. J. Am. Chem. Soc. 140, 9854-9867 (2018).

38. Bie, X., Kubota, K., Hosaka, T., Chihara, K. \& Komaba, S. A novel K-ion battery: hexacyanoferrate(ii)/graphite cell. J. Mater. Chem. A 5, 4325-4330 (2017).

39. Zhang, W., Pang, W. K., Sencadas, V. \& Guo, Z. Understanding High-Energy-Density Sn4P3 Anodes for Potassium-Ion Batteries. Joule 2, 1534-1547 (2018).

40. Jian, Z., Xing, Z., Bommier, C., Li, Z. \& Ji, X. Hard Carbon Microspheres: Potassium-Ion Anode Versus Sodium-Ion Anode. Adv. Energy Mater. 6, 1-5 (2016).

41. Homer, J. Solvent Effects on Nuclear Magnetic Resonance Chemical Shifts. Applied Spectroscopy Reviews 9, (1975).

42. Teijiro Yonezawa, Isao Morishima, K. T. Solvent Effects in NMR Spectroscopy. I. Chemical Shifts Induced by the Addition of Protic Substances to Benzene Solutions of Several Polar Compounds. Bull. Chem. Soc. Jpn. 40, 1807-1813 (1967).

43. Freunberger, S. A. et al. Reactions in the rechargeable lithium-02 battery with alkyl carbonate electrolytes. J. Am. Chem. Soc. 133, 8040-8047 (2011).

44. Marcus, Y. Thermodynamic functions of transfer of single ions from water to nonaqueous and mixed solvents: Part 3 - Standard potentials of selected electrodes. Pure Appl. Chem. 57, 11291132 (2007).

45. May, R., Zhang, Y., Denny, S. R., Viswanathan, V. \& Marbella, L. E. Leveraging Cation Identity to Engineer Solid Electrolyte Interphases for Rechargeable Lithium Metal Anodes. Cell Reports Phys. Sci. 1, 100239 (2020).
46. Groß, U., Rüdiger, S., Grimmer, A. R. \& Kemnitz, E. 19F-NMR solid state investigations of monovalent alkali metal fluorides and tetraalkylammonium fluorides. J. Fluor. Chem. 115, 193-199 (2002).

47. Clément, R. J., Kitchaev, D., Lee, J. \& Gerbrand Ceder. Short-Range Order and Unusual Modes of Nickel Redox in a Fluorine-Substituted Disordered Rocksalt Oxide Lithium-Ion Cathode. Chem. Mater. 30, 6945-6956 (2018).

48. Shchukarev, A. V. \& Korolkov, D. V. XPS study of group IA carbonates. Cent. Eur. J. Chem. 2, 347362 (2004).

49. Randles, J. E. B. Kinetics of Rapid Electrode Reactions. Discuss. Faraday Soc. 1, 11-19 (1947).

50. R. Lowndes \& Martin, D. Dielectric Constants of Ionic Crystals and Their Variations With Temperature and Pressure. 316, 351-375 (1970).

51. Wakamura, K. Effects of electronic band on activation energy and of effective charge on lattice distortion in superionic conductors. $J$. Phys. Chem. Solids 59, 591-598 (1998).

52. Yildirim, H., Kinaci, A., Chan, M. K. Y. \& Greeley, J. P. First-Principles Analysis of Defect Thermodynamics and Ion Transport in Inorganic SEI Compounds: LiF and NaF. ACS Appl. Mater. Interfaces 7, 18985-18996 (2015).

53. He, M., Guo, R., Hobold, G. M., Gao, H. \& Gallant, B. M. The intrinsic behavior of lithium fluoride in solid electrolyte interphases on lithium. Proc. Natl. Acad. Sci. U. S. A. 117, 73-79 (2020).

54. Okuno, Y., Ushirogata, K., Sodeyama, K. \& Tateyama, Y. Decomposition of the fluoroethylene carbonate additive and the glue effect of lithium fluoride products for the solid electrolyte interphase: An ab initio study. Phys. Chem. Chem. Phys. 18, 8643-8653 (2016).

55. Nie, M. \& Lucht, B. L. Role of Lithium Salt on Solid Electrolyte Interface (SEI) Formation and Structure in Lithium Ion Batteries. J. Electrochem. Soc. 161, A1001-A1006 (2014).

56. Eshetu, G. G. et al. In-Depth Interfacial Chemistry and Reactivity Focused Investigation of LithiumImide- and Lithium-Imidazole-Based Electrolytes. ACS Appl. Mater. Interfaces 8, 16087-16100 (2016).

57. Terborg, L. et al. Ion chromatographic determination of hydrolysis products of hexafluorophosphate salts in aqueous solution. Anal. Chim. Acta 714, 121-126 (2012). 


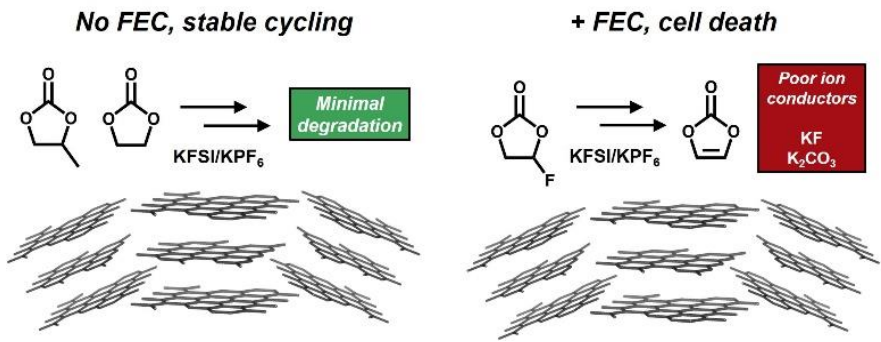

